\title{
A NUMERICAL INVESTIGATION OF A SHOCK-TUBE-DRIVEN CONDUCTIVITY CHANNEL
}

\author{
B. L. Venable *, D. A. Anderson ${ }^{\dagger}$, and D. R. Wilson $\ddagger$ \\ The University of Texas at Arlington, Arlington, Texas
}

\begin{abstract}
$\underline{\text { Abstract }}$
A code using the MacCormack scheme modified to be TVD has been written to analyze the flow in a magnetohydrodynamic conductivity channel driven by a reflected shock tube with a heated driver. Items considered include the thermodynamic and electrical properties of the potassium-seeded plasma, both with and without a current applied along the flow, and the steady-state test time in the channel. An inviscid, quasi-one-dimensional model was used with an electric power flux term included in the energy equation to account for the energy addition of the applied current. It was determined that, as a consequence of the high temperature ratios across the primary diaphragm in the shock tube, no steady-state window exists in the channel. Conductivity channel investigations revealed that Joule heating effects begin at a channel current of two Amperes and become significant above a current of ten Amperes.
\end{abstract}

\section{Introduction}

Recently, attention has been refocused on magnetohydrodynamics (MHD) in an effort to produce a facility for conducting large scale hypersonic propulsion testing. Studies were initially focused on the development of an unseeded, nonequilibrium accelerator to provide clean air test conditions. ${ }^{1,2}$ However, efforts are now being directed toward the more readily attainable goal of a seeded, equilibrium accelerator. As a participant in the preliminary studies for this project, The Department of Mechanical and Aerospace Engineering at The University of Texas at Arlington (UTA) is developing a proposal for a plan to augment the existing hypersonic shock tunnel on campus with an MHD accelerator. Initial

\footnotetext{
*Graduate Student, Member AIAA

†Professor, Aerospace Engineering Program, Associate Fellow AIAA

†Director, Aerospace Engineering Program, Associate Fellow AIAA
}

Copyright (C) 1996 by the American Institute of Aeronautics and Astronautics, Inc. All rights reserved. studies would involve the design and construction of a Hall configured conductivity channel with no applied magnetic field in which an appraisal of the conductivities, voltages, currents, and thermodynamic properties can be conducted. Ideally, the program would then progress to the construction and testing of a fully operational accelerator.

The successful implementation of the conductivity channel tests in the UTA hypersonic shock tunnel facility should be supported by computational data to provide preliminary information about the steady-state test window and the thermodynamic conditions in the channel. A sketch of a typical reflected shock tunnel and wave diagram is shown in Figure 1. The wave diagram indicates a lim-
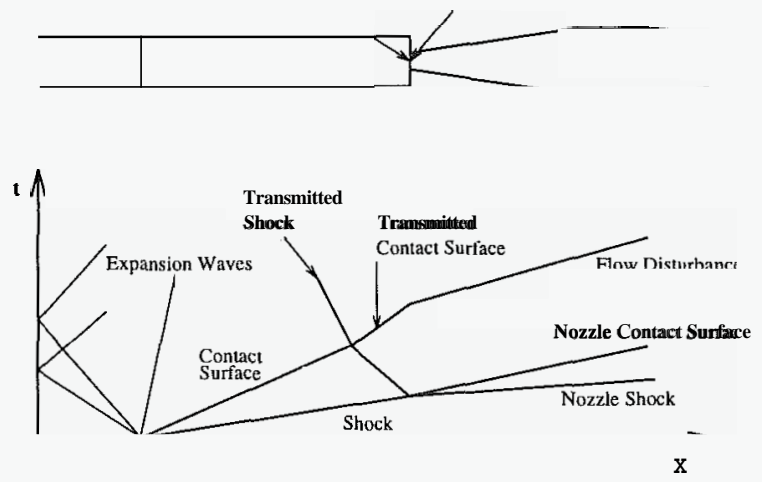

Figure 1: Typical hypersonic shock tunnel with wave diagram

ited test window between the passage of the nozzle contact surface and the flow disturbance due to the passage of the transmitted contact surface. A transient, quasi-one-dimensional code has been developed to investigate this test window and to determine the thermodynamic and electrical properties in the conductivity channel. Included in the code are an equilibrium air model and an electrical conductivity model. Once steady state is reached in the channel, a pulse electric current is applied to the seeded air in the direction of the flow. Changes in the thermodynamic properties and electrical con- 
ductivity take place as a result of the energy addition to the flow. These changes, along with the voltage distribution in the channel, are investigated. A seed species of one percent potassium carbonate is used to facilitate ionization in the channel.

\section{$\underline{\text { Computational Method }}$}

The quasi-one-dimensional set of equations may be written as

$$
\frac{\partial \mathbf{U}}{\hat{a} t}+\frac{\partial \mathbf{F}}{\partial x}=\mathbf{S}
$$

The vector of conserved variables is

$$
\mathbf{U}=\left(\begin{array}{c}
\rho A \\
\rho u A \\
E_{T} A
\end{array}\right)
$$

where $E_{T}=p\left(e+1 / 2 u^{2}\right), A$ is the cross-sectional area, and $p, u$, and $e$ are the density, velocity, and internal energy per unit mass, respectively. The flux vector is

$$
\mathbf{F}=\left(\begin{array}{c}
\rho u A \\
\left(\rho u^{2}+p\right) A \\
\left(E_{T}+p\right) \rho u A
\end{array}\right)
$$

where $\mathrm{p}$ is the static pressure. Source terms accounting for the varying area and the MHD effects are contained in the vector $\mathbf{S}$ as given below.

$$
\mathbf{S}=\left(\begin{array}{c}
0 \\
p \cdot d A / d x \\
J_{x} E_{x}
\end{array}\right)
$$

Here $J$ is the current density magnitude and $E$ is the magnitude of the electric field.

The governing equations were solved using an explicit MacCormack predictor-corrector method. A post-processor step was included to make the scheme total variation diminishing. Implementation of this scheme followed the presentation by Yee. ${ }^{3}$ The equilibrium air equation of state was provided by the TGAS subroutines of Srinivasan, Tannehill, and Weilmuenster. ${ }^{4}$ Electrical conductivities were calculated using a version of the NASA CEC $\operatorname{code}^{5} \bmod -$ ified to provide conductivity data. A database of conductivities was constructed over a span of temperatures and pressures covering the operating range of the conductivity channel. Then, given a temperature and pressure, the corresponding value of the conductivity was found by interpolation.

\section{Nozzle Starting Process}

A study of the starting process in a hypersonic nozzle was performed by Smith.' His results are used as a benchmark for the time accuracy of the transient nozzle flow. As the secondary diaphragm breaks behind the reflected shock in the driven tube, a flow resembling that in Figure 2 develops in the nozzle. Initially, a shock wave followed by a contact surface and an unsteady expansion fan move through the nozzle. The increase in area of the nozzle causes the primary shock to decelerate as it progresses. This has the effect of causing the $u-c$ characteristics behind the primary shock to rotate progressively counterclockwise. Eventually these Characteristics converge and form a secondary shockjust behind the contact surface. As this shock structure moves down the nozzle the secondary shock gains strength and lags farther behind the primary shock. The nozzle starting time, then, may be determined by either the passage of the shock structure or the unsteady expansion fan. The dominating phenomenon depends on the nozzle geometry and the starting conditions.
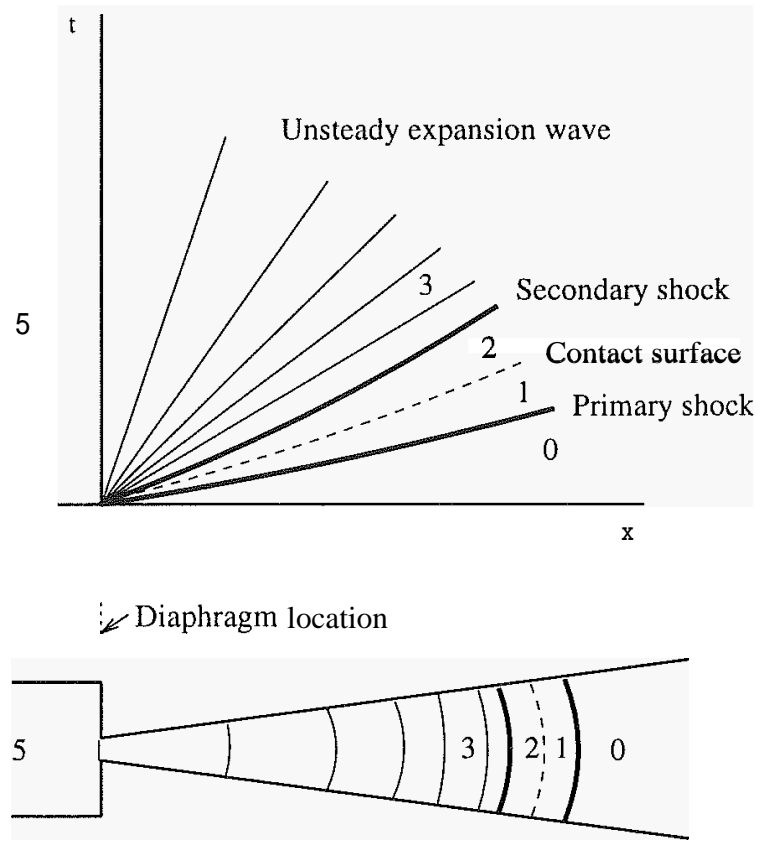

Figure 2: Characteristic diagram of nozzle start

Results produced by this code are compared with experimental and method of characteristics data published by Smith in Figure 3. There is excellent agreement with the method of characteristics data, as the slopes of the curves agree within approximately three percent. The difference between the numerical and experimental shock trajectories is attributed by Smith to the effect of smoothness of curvature on the shock speed. The superior smoothness of the actual facility compared to that of the 
discrete geometry permits the starting shock waves to pass more quickly.

The foundation has been set for the calculation of the transient and steady-state conditions in the accelerator channel.

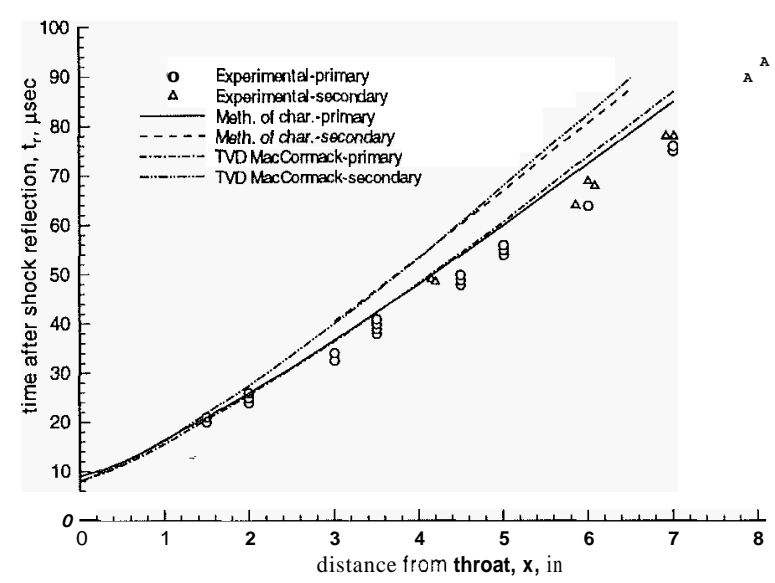

Figure 3: Nozzle starting shock trajectories

\section{The Conductivitv Channel}

The conductivity channel under consideration is of circular cross section with a design Mach number of 1.6. A sketch of the channel is shown in Figure 4. Note that this drawing is not to scale. Of the many possible electrode arrangements, the most likely candidate consists of 22 electrodes and 21 insu-
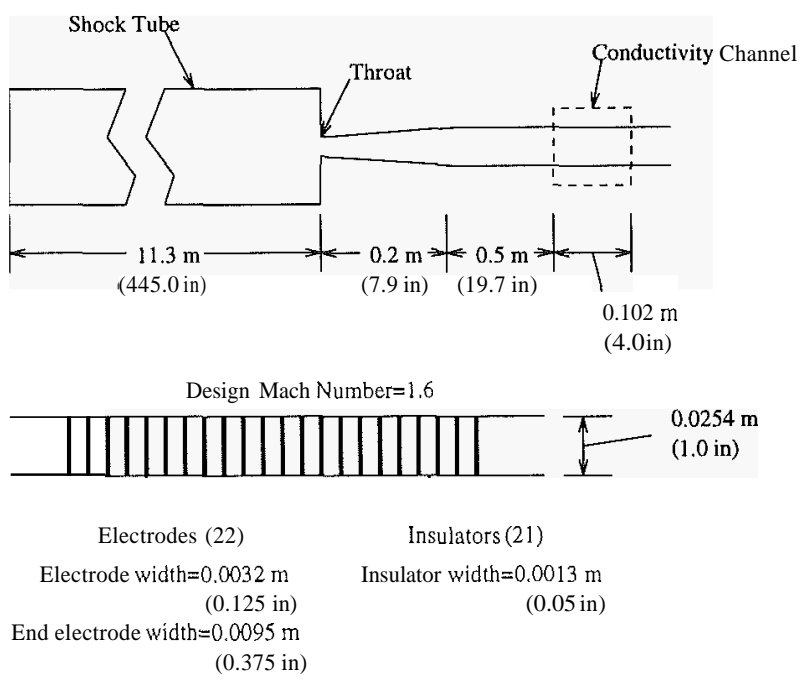

Figure 4: Sketch of conductivity channel lators for a total length of $0.1 \mathrm{~m}$ (4in). A conical nozzle with a divergence half-angle of 0.45 degrees expands the flow from the throat to the channel. The shock tube consists of a $3 \mathrm{~m}(10 \mathrm{ft})$ long driver section and a $8.3 \mathrm{~m}(27 \mathrm{ft})$ long driven section, both of which have a $0.152 \mathrm{~m}(6 \mathrm{in})$ inner diameter. It is desired to gather information for channel static pressures ranging from $0.101 \mathrm{MPa}$ (latm) to $10.1 \mathrm{MPa}$ (100atm) and temperatures from $2000 \mathrm{~K}$ to $4000 \mathrm{~K}$. A heated driver is necessary to achieve these conditions in the conductivity channel. A reflected detonation wave will be used to produce the heated driver. The ignition will occur in the driver tube at the end opposite the primary diaphragm. A detonation wave will then travel down the driver and reflect off the primary diaphragm. The high enthalpy conditions behind this reflected detonation wave will drive the subsequent process. Numerically, this will be simulated by specifying the conditions throughout the driver section equal to those behind the reflected detonation wave. Any interactions in the driver occurring after the reflection of, and due to, the detonation wave are not included in this analysis. However, these interactions will not effect the channel flow during the time frame of importance. It is assumed throughout this analysis that the driver section contains air.

Driver section conditions are listed in Table 1 along with the resulting stagnation conditions in region 5 behind the reflected shock wave at the end of the driven tube. In all cases the driven tube and nozzle were initialized to $0.101 \mathrm{MPa}$ (latm) and $300 \mathrm{~K}$.

Table 1:

Shock tube starting conditions

\begin{tabular}{|c|c|c|c|}
\hline Run No. & 1 & 2 & 3 \\
\hline \hline$p_{4}$ & $399 \mathrm{~atm}$ & $195 \mathrm{~atm}$ & $46 \mathrm{~atm}$ \\
\hline$T_{4}$ & $4500 \mathrm{~K}$ & $4320 \mathrm{~K}$ & $4000 \mathrm{~K}$ \\
\hline$p_{5}$ & $422 \mathrm{~atm}$ & $271 \mathrm{~atm}$ & $90 \mathrm{~atm}$ \\
\hline$T_{5}$ & $4210 \mathrm{~K}$ & $3342 \mathrm{~K}$ & $1832 \mathrm{~K}$ \\
\hline
\end{tabular}

\section{$\underline{\text { Results }}$}

\section{Channel Test Window}

The first order of business was to determine the time range of steady-state conditions in the conductivity channel. Figures 5, 6, and 7 show time traces at the entrance and exit of the channel for high, 
medium, and low driver pressures, respectively. The passage of the nozzle starting elements is easily identified. Due to the short length and small area ratio (1.25) of the nozzle, the primary nozzle starting shock, nozzle contact surface, and secondary starting shock are closely spaced. These are followed by the unsteady expansion wave. In all three cases the flow disturbance caused by the transmitted contact surface occurs before the complete passage of the unsteady expansion: No steady-state run time exists in the channel at the expected test pressures and temperatures. In the high pressure case a quasisteady-state condition exists at a lower temperature and pressure after the passage of the transmitted contact surface; however, the gas in the channel at this point contains detonation products and will not exhibit true-air chemistry. Even this does not exist with a low pressure driver.
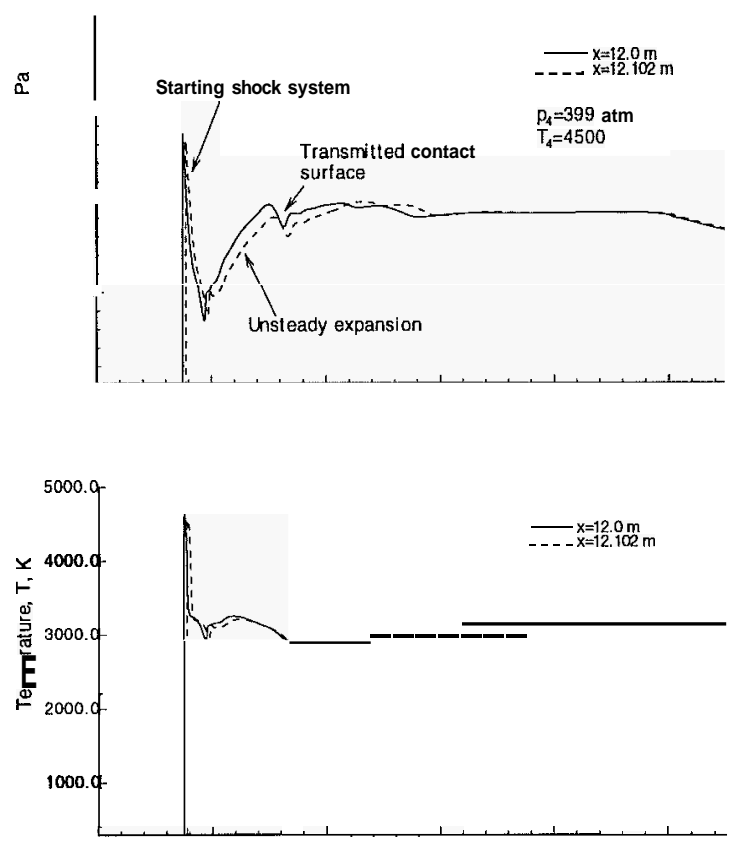

Figure 5: Time traces for high driver pressure and temperature

There are two reasons for the lack of test time in the conductivity channel. First, with such extreme temperature ratios across the primary diaphragm, the contact surface follows very closely behind the shock traveling into the driven tube. Just before shock reflection 'the contact surface is approximately $1 \mathrm{~m}$ behind the shock for the $4500 \mathrm{~K}$ driver, whereas the contact surface trails the shock by $2 \mathrm{~m}$ for a non- heated driver at the same pressure. This reduces significantly the time required for the passage of the transmitted contact surface through the channel. A possibility for delaying the passage of the transmitted contact surface is the "slug stretcher" developed at AEDC. ${ }^{7}$ This is an area contraction that is fitted on the end of the driven tube upstream of the throat which can increase the steady run time of the shock tunnel.
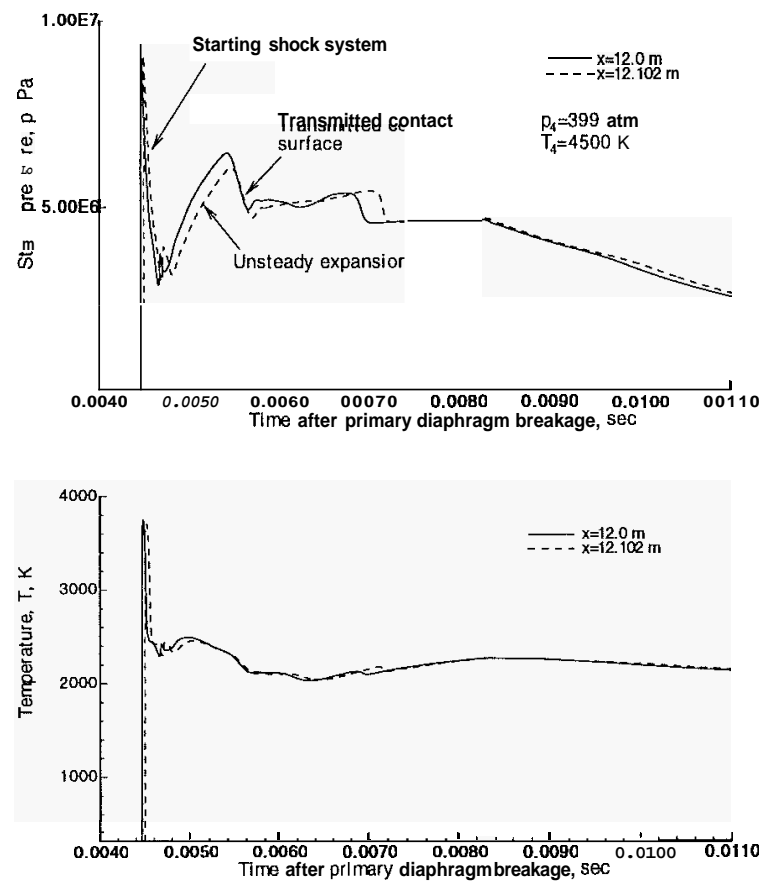

Figure 6: Time traces for medium driver pressure and temperature

A second factor is the duration of the nozzle starting process. This is controlled both by the nozzle geometry and the starting conditions. It was discussed previously how the starting process can be dominated by either the shock structure (secondary shock) or the unsteady expansion wave. It is obvious that with this case the nozzle start is delayed by the unsteady expansion. It has been shown by Stalker and Mudford ${ }^{8}$ that for a nozzle with a given length and area ratio initialized with a stationary gas, the speed of the unsteady expansion increases and the speed of the secondary shock decreases as the area increases. It is then possible to decrease the residence time of the unsteady expansion in the conductivity channel by increasing the area ratio of the nozzle while maintaining the length. However, this would increase the Mach number in the chan- 
nel and provide lower channel static pressures and temperatures.

Another possibility for decreasing the time to start the conductivity channel is to lower the initial channel pressure. Smith' states that nozzle initial pressures less than the steady-flow static pressure do not result in any significant increase in test time if the stagnation temperature is less than $3500 \mathrm{~K}$. The stagnation temperatures here range from $4000 \mathrm{~K}$ to $4500 \mathrm{~K}$. The nozzle initial pressure here is two orders of magnitude less than the steady-flow static pressure for the high driver pressure and one order of magnitude less for the low driver pressure. Calculations showed that lowering the channel initial pressure further had no effect on the starting process.

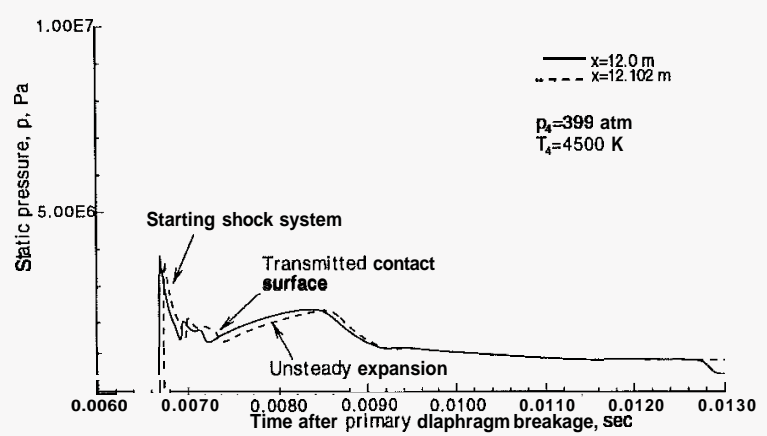

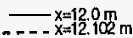

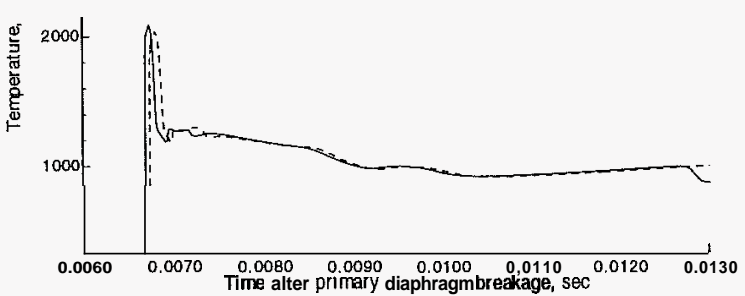

Figure 7: Time traces for low driver pressure and temperature

\section{Current Application}

The quasi-steady-state flow between $t=$ $6.5 \mathrm{msec}$ and $7.5 \mathrm{msec}$ in Figure 5 was chosen to pass a current through the conductivity channel. Although this window does not occur at the expected channel conditions due to the premature passage of the transmitted contact surface, the pressure and temperature are suitable for the gathering of useful information concerning the response to a current passed through the channel. The gas in the channel during this time frame is assumed to be air.

Currents ranging from $1.0 \mathrm{~mA}\left(\mathrm{~J}=2.0 \mathrm{~A} / \mathrm{m}^{2}\right)$ to 40.0A $\left(J=80,000 \mathrm{~A} / \mathrm{m}^{2}\right)$ were passed through the channel. The resulting $V^{-} I$ plot is shown in Figure 8. The curve is linear below $I=2.0 \mathrm{~A}$ indicating a negligible change in the temperature of the plasma due to the current application. Between $I=2.0 \mathrm{~A}$ and $I=10.0 \mathrm{~A}$ the effects of Joule heating begin to be observed. Above $I=10.0 \mathrm{~A}$ the Joule heating is such a large factor that any increase in the current does not result in a significant voltage increase; in fact, the voltage begins to decrease slightly above $I=30.0$ A

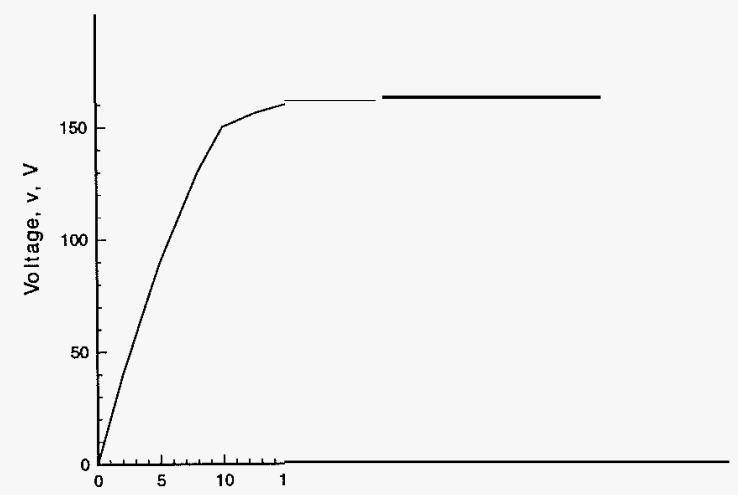

Figure 8: Conductivity channel $\mathrm{V}$-I diagram

Currents of 2.0A $\left(\mathrm{J}=4000 \mathrm{~A} / \mathrm{m}^{2}\right), 15.0 \mathrm{~A}(\mathrm{~J}=$ $\left.30,000 \mathrm{~A} / \mathrm{m}^{2}\right)$, and $30.0 \mathrm{~A}\left(J=60,000 \mathrm{~A} / \mathrm{m}^{2}\right)$ were chosen for further investigation. These currents correspond respectively to the linear portion of the $V$-I curve, the portion where significant Joule heating begins, and the portion where Joule heating dominates. The applied current pulse was shaped as a square wave with faired edges to help reduce oscillations caused by a discontinuous application and removal. In all cases the current pulse lasted from $t=6.5 \mathrm{msec}$ to $t=7.5 \mathrm{msec}$ after primary diaphragm breakage with the application and removal being smoothed over $0.1 \mathrm{msec}$ each. The resulting voltages across the conductivity channel for the three mentioned currents are shown as functions of time in Figure 9. With $I=2.0 \mathrm{~A}$ the voltage is relatively constant throughout the duration. The slight wave motion is most likely due to the quick current application. If the current were left applied for a long period of time this would likely dissipate. Effects of Joule heating can be seen at at $I=15.0 \mathrm{~A}$ and, more significantly, $I=30.0 \mathrm{~A}$. In both cases the 
voltage increases rapidly toward that which would be attained in the absence of Joule heating. Before it reaches that value, the conductivity of the gas increases with the temperature and the voltage drops to the quasi-steady value. Joule heating occurs quickly, as in both cases the voltage begins to drop before the current reaches the full magnitude at $t=6.6 \mathrm{msec}$.

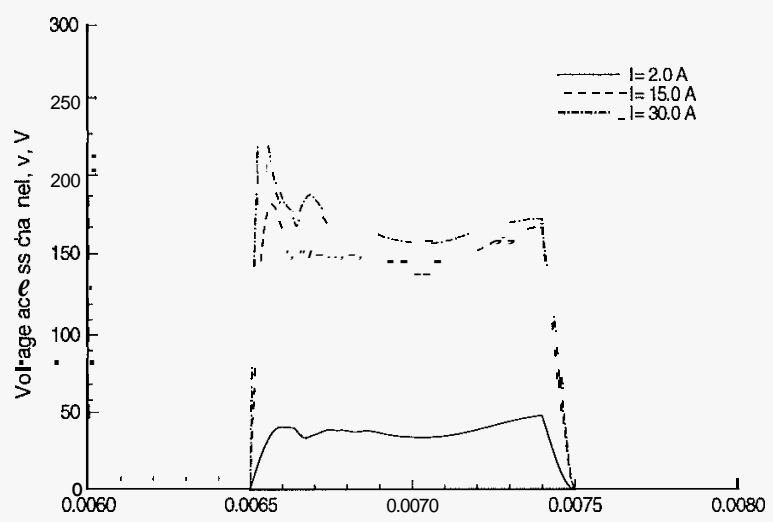

Figure 9: Channel voltages

Details of the channel conditions that are of interest include the changes in the pressure, temperature, and conductivity with current application and the voltage distribution along the channel. Figures 10,11 , and 12 depict these items for $I=2.0 \mathrm{~A}$. Time traces of pressure and temperature in Figure 10 clearly show the current application and the resulting oscillations. A spike in pressure and temperature occurs as the current reaches $I=2.0 \mathrm{~A}$ at $t=6.6 \mathrm{msec}$. This spike then quickly passes out of the channel. The pressures and temperatures at the entrance and exit of the channel remain almost equal. This is an indication of the lack of Joule heating. Plots of the temperature and conductivity along the channel at various times during and around current application are shown in Figure 11. It can be seen that the conductivity varies with the temperature. Neither the temperature nor the conductivity change significantly. Figure 12 shows an almost linear voltage drop along the channel for this case. This is a result of the relatively constant conductivity through the channel.

Similar plots are included for $I=30.0 \mathrm{~A}$ in Figures 13 to 15 . The presence of Joule heating is obvious in the time traces as both the pressure and temperature rise significantly. It is worth pointing out that the pressure and temperature remain nearly constant at the inlet of the channel. As the gas proceeds through the channel and is subjected to the current, it becomes energized and reaches the peak values at the exit. Both the temperature and pressure increase quickly with application of the current and decrease quickly as the current diminishes. Voltage drops along the length of the channel for these currents increase in curvature due to changes in the conductivity with position in the channel. The highest voltage drops occur in the upstream portion of the channel where the conductivity is lowest.

$\mathrm{John}^{\mathrm{g}}$ states that that minimum conductivities of $10.0 \mathrm{~S} / \mathrm{m}$ are required to successfully operate a MHD accelerator. At these channel conditions, $9.8 \mathrm{MPa}$ and $3100 \mathrm{~K}$ before current application, the conductivity is approximately $10.0 \mathrm{~S} / \mathrm{m}$. With the application of a large current and the resulting contribution of the Joule heating, the conductivities increase to almost $70.0 \mathrm{~S} / \mathrm{m}$. It seems likely that the Joule heating can aid in the ionization of the gas and eliminate the necessity to produce extremely high static conditions in the channel beforehand.

\section{$\underline{\text { Conclusions }}$}

It was determined that no steady test window exists in the conductivity channel for the starting conditions and geometry being considered. This is due primarily to the close proximity of the contact surface to the shock wave at the time of reflection of the shock in the driven tube. An increase in the channel Mach number is one possible solution. It is unknown whether or not this would decrease the nozzle starting time sufficiently to provide a steady-state test window in the channel. It would, however, reduce the channel static pressure and temperature. Channel conductivity tests revealed that conductivities of $\sigma=10.0 \mathrm{~S} / \mathrm{m}$ are present in the chaiinel even at static conditions lower than those originally planned. One other idea is the slug stretcher. Implementation of this device could result in an acceptable channel test window at static conditions near those originally expected.

Results of the electrical calculations in the conductivity channel showed all the correct trends. Electrical results can not be validated until experimental work is accomplished; however, the data indicates that Joule heating becomes a significant phenomenon above currents of $I=10.0 \mathrm{~A}$. The resulting temperature rise might possibly be advantageous in that it could help reduce the magnitude of the static conditions that must be initially produced in the conductivity channel, thereby allowing a higher 
channel Mach number as a method of producing a steady test time.

One aspect of the flow that was neglected in this analysis is the influence of viscosity. It is believed that because of the short length of the nozzle and conductivity channel, boundary layer formation will not be sufficient to appreciably affect the solution. The strong expansion taking place in the nozzle will also inhibit the development of the boundary layer.

The data contained herein should be useful in the development of the experimental facility. Although a problem with the available test time was revealed, it is hoped that clues to a satisfactory solution were also presented.

\section{Acknowledgment}

This work was conducted as part of the NASA/UTA Center for Hypersonic Research, and was supported by NASA Office of Aeronautics under NASA Grant NAGW-3714, monitored by Dr. Isaiah Blankson.

\section{References}

[1] Simmons, G., Nelson, G., Hiers, R., and Western, A., "An Unseeded Air MHD Accelerator Concept for High Mach Number Hypersonic Propulsion," AIAA Paper 89-2535, July 1989.

[2] Simmons, G. A., Nelson, G. L., and Lee, Y. M., "Analysis of An Unseeded, Nonequilibrium MHD Accelerator Concept For Hypersonic Propulsion Ground Testing," AIAA Paper 923994, July 1992.
[3] Yee, H. C., "Upwind and Symmetric ShockCapturing Schemes," NASA TM 89464, Ames Research Center, Moffet Field, CA, May 1987.

[4] Srinivasan, S., Tannehill, J. C., and Weilmuenster, K. J., "Simplified Curve fits for the Thermodynamic Properties of Equilibrium Air," NASA Reference Publication 1181, 1987.

[5] Gordon, S. and McBride, B. J., ('Computer Program for Calculation of Complex Chemical Equilibrium Compositions, Rocket Performance, Incident and Reflected Shocks, and Chapman-Jouguet Detonations," NASA SP273, NASA Lewis Research Center, Cleveland OH, March 1976.

[6] Smith, C. E., "The starting process in a hypersonic nozzle," Journal of Fluid Mechanics, Vol. 24, April 1966, pp. 625-640.

[7] Norfleet, G. D., “Theoretical and Experimental Study of a Device for Increasing the Uncontaminated Flow Time of a Reflected Shock Tunnel," AEDC-TR-67-172, Arnold Engineering Development Center, Arnold AFB T N, October 1967.

[8] Stalker, R. J. and Mudford, N. R., "Unsteady shock propagation in a steady flow nozzle expansion," Journal of Fluid Mechanics, Vol 241, 1992, pp. 525-548.

[9] John, J. E. A., Gas Dynamics, 2nd ed., Allyn and Bacon, Inc., Boston, MA, 1984. 

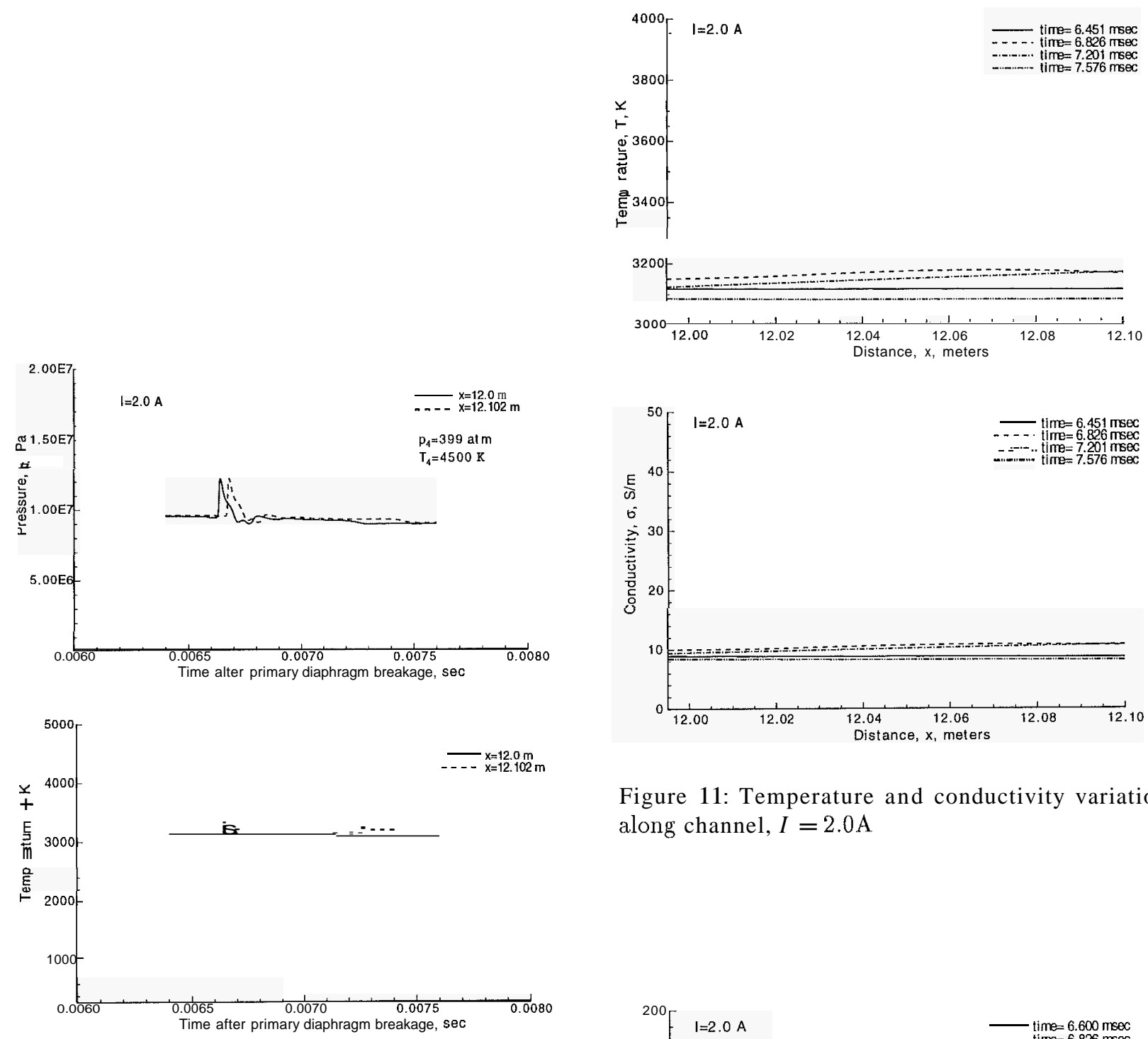

Figure 11: Temperature and conductivity variation along channel, $I=2.0 \mathrm{~A}$

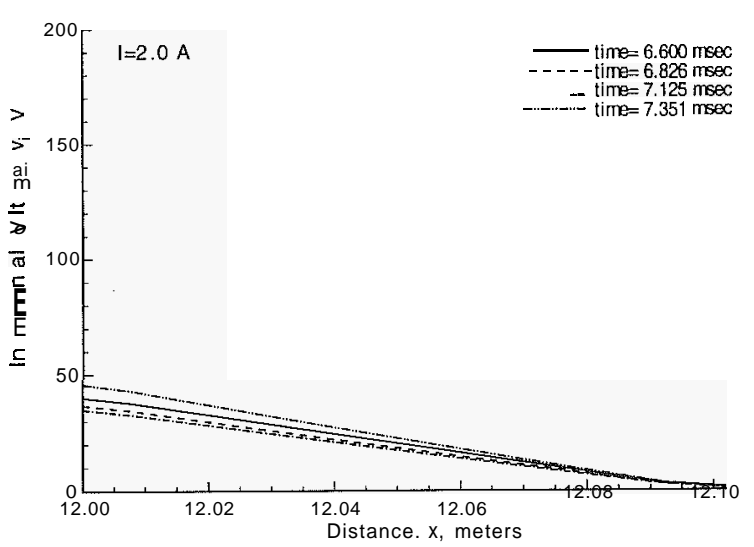

Figure 12: Voltage drop along channel, $I=2.0 \mathrm{~A}$ 

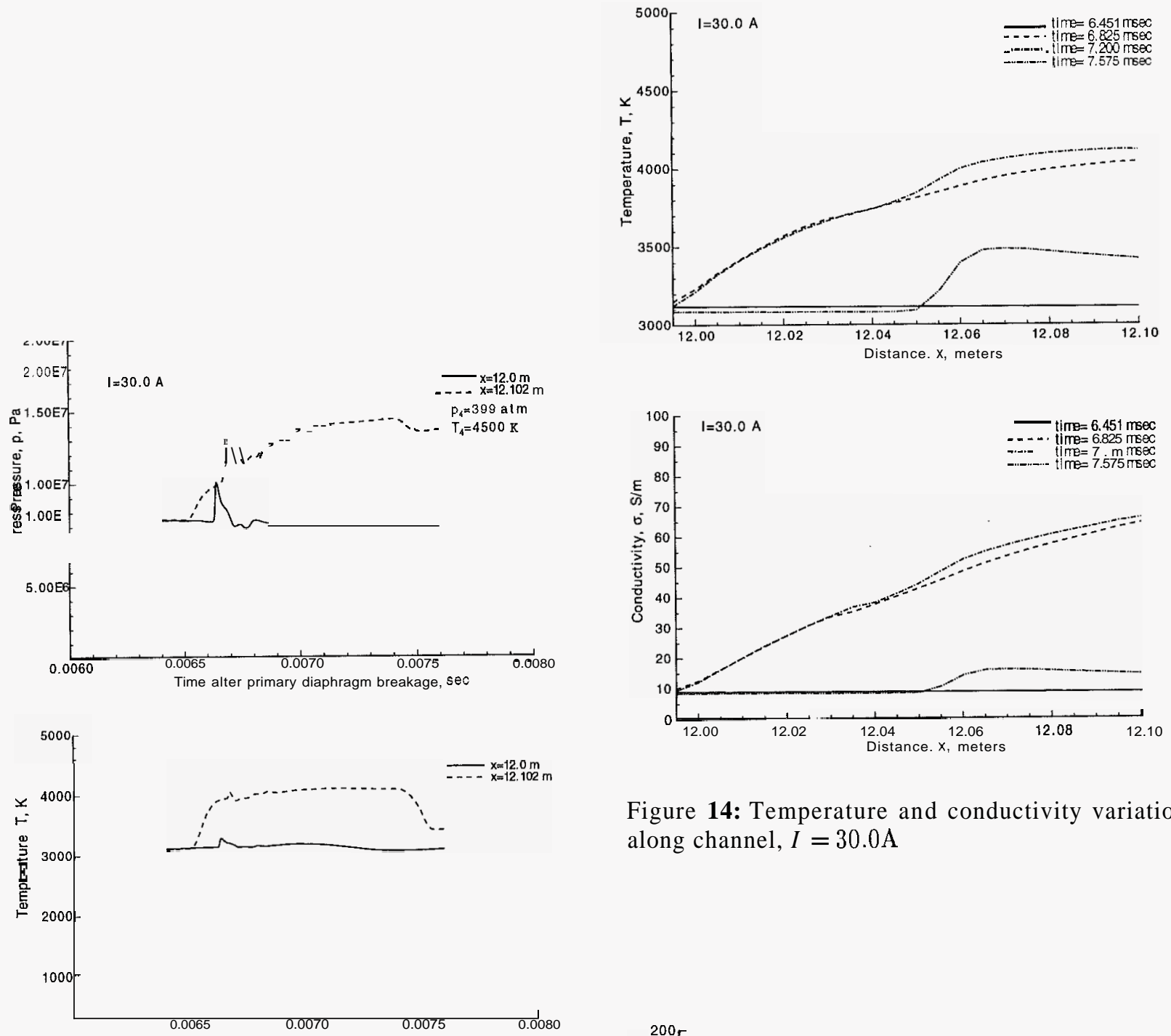

Figure 14: Temperature and conductivity variation along channel, $I=30.0 \mathrm{~A}$

Figure 13: Time traces for $I=30.0 \mathrm{~A}$

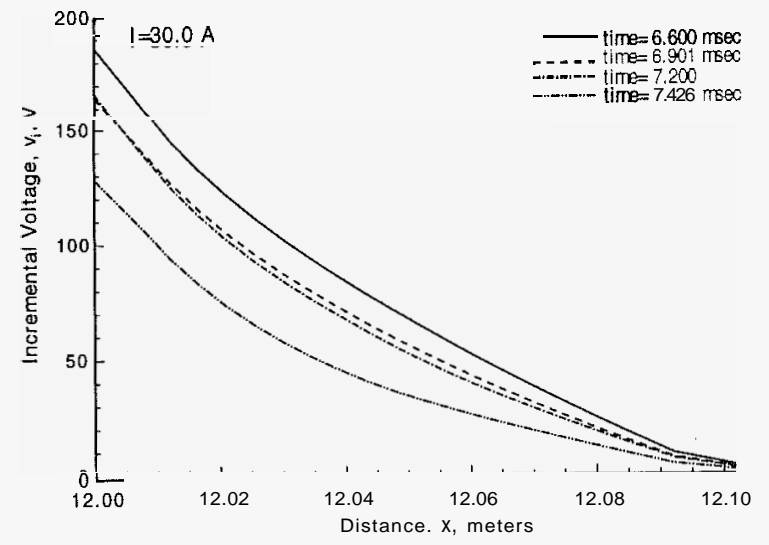

Figure 15: Voltage drop along channel, $I=30.0 \mathrm{~A}$ 